\title{
Non-linear parametric resonance driven oscillations of dumbell satellite in elliptical orbit under the combined effects of magnetic field of the earth and oblateness of the earth
}

\author{
A. Narayan *, M. D. Pandey \\ Department of Mathematics, Bhilai Institute of Technology Bhilai House Durg, India \\ *Corresponding author E-mail: ashutoshmaths.narayan@gmail.com
}

\begin{abstract}
Parametric resonance driven oscillations of a dumbbell satellite in elliptical orbit in central gravitational field of force under the combined effects of perturbing forces Earth Magnetic field and Oblateness of the Earth has been studied. The system comprises of two satellite connected by a light, flexible and inextensible cable, moves like a dumbbell satellite in elliptical orbit, in central gravitational field of force. The gravitational field of the Earth is the main force governing the motion and magnetic field of the Earth and Oblateness of the Earth are considered to be perturbing forces, disturbing in nature. Non-linear oscillations of dumbbell satellite about the equilibrium position in the neighborhood of parametric resonance $\omega=\frac{1}{2}$, under the influence of perturbing forces, which is suitable for exploiting the asymptotic methods of Bogoliubov, Krilov and Metropoloskey has been studied, considering 'e' to be a small parameter. The Hamiltonian has been constructed for the problem and phase analysis has been applied to investigate the stability of the system.
\end{abstract}

Keywords: Evolutional and Non-Evolutional; Perturbing Forces; Stability.

\section{Introduction}

This paper is devoted to the analysis of non-linear parametric resonance driven oscillations of cable connected satellites system in elliptical orbit connected by a light, flexible and inextensible cable moving in the central gravitational field of the Earth under the combined effects of the Earth magnetic field and Oblateness of the Earth. The satellites are considered to be charged material particle and the motion of the system is studied relative to their centre of mass, under the assumption that the later moves along elliptical orbit. The cable connecting the two satellites is taut and nonelastic in nature such that, the system moves like a dumbbell satellite. Many space configurations of cable connected satellite system have been proposed and analysed by different authors like two satellite are connected by a rod (Celletti et al 2008), two or more satellites are connected by a tether (M. Krupa et al $2000 \& 2006$ ), (Beletsky \& Levin 1993), (Mishra \& Modi 1982). All these authors have mentioned numerous important applications of system and stability of relative equilibrium, if the system moves in a circular and elliptical orbit. (Beletsky \& Novikova 1969), studied the motion of a system of two satellite connected by a light, flexible and inextensible string in the central gravitational field of force relative to their centre of mass, which is itself assumed to more along a Keplerian elliptical orbit under the assumption that the two satellite are moving in the plane of the centre of mass. The same problem in its general form, was further investigated (Singh 1971,
1973), these works conducted the analysis of relative motion of the system for the elliptical orbit of the centre of mass in the two dimensional as well as three dimensional cases. (Narayan \& Singh 1987, 1990, 1992), studied non-linear oscillations due to solar radiation pressure of the centre of mass of the system moves along an elliptical orbit.

The different aspects of the problem of stability of satellites in low and high altitude orbit with different perturbation forces are studied by many scientists, (Sharma \& Narayan 2001,2002), (Singh et al 1971,1973,1997), (Das et al 1976), and (Narayan et al 1987,1990,1992). Special references are mentioned (Sarychev et al 2000, 2007) studied the problem determining all equilibria of a satellite subject to gravitational and aerodynamic torque in circular orbit. All bifurcation values of the parameter corresponding to qualitative changes of stability domain are determined. (Palacian 2007), studied the dynamics of a satellites orbiting are Earth like planet at low altitude orbit and perturbation is caused by inhomogeneous potential due to the Earth. (Langbort 2002), studied bifurcation of relative equilibria in the main problem of artificial satellite theory for a prolate body. (Markeev et al 2003), studied the planar oscillation of a satellite in a circular orbit. (Ayub Khan et al 2011), investigated chaotic motion in problem of dumbbell satellite.

The present paper deals with the non-linear parametric driven oscillation of dumbbell satellite in elliptical orbit under the combined effects of magnetic field of the Earth and oblateness of the 
Earth. The perturbing forces due to Earth magnetic field results from the interaction between space craft's residual magnetic field and the geomagnetic field. The perturbing force is arising due to magnetic moments, eddy current and hysteresis, out of these the space craft magnetic moment is usually the dominant source of disturbing effects.

\section{Equation of motion}

The combined effects of the geomagnetic field and Oblateness of the Earth on the motion and stability of the satellite connected by a light, flexible and inextensible cable, under the influence of the central gravitational field of the Earth have been considered. The analysis of Evolutional and Non-evolutional motion of dumbell satellite in elliptical orbit has been restricted to two

Dimensional case, we have assumed that the satellites are moving in the orbital plane of the centre of mass of the system. The motion and stability of cable connected satellite system under the effects of Earth's magnetic field, (Das et al 1976), (Narayan et al 2004), and combined effects of Earth magnetic field and oblateness of the Earth, (Narayan and Pandey 2010), in elliptical and in low altitude orbit has been studied. The equation of two dimensional motion of one of the satellite under the rotating frame of reference in (Nechville's 1926) co-ordinate system, relative to their centre of mass, which moves along equatorial orbit under the combined influence of the Earth magnetic field and Oblateness of the Earth can be represented in (2.1):

$$
\begin{aligned}
& x^{\prime \prime}-2 y^{\prime}-3 \rho x=\lambda_{\alpha} x+\frac{4 A x}{\rho}-\frac{B}{\rho} \cos \delta \\
& y^{\prime \prime}-2 x^{\prime}=\lambda_{\alpha} y-\frac{A y}{\rho}-\frac{B \rho^{\prime}}{\rho^{2}} \cos \delta .
\end{aligned}
$$

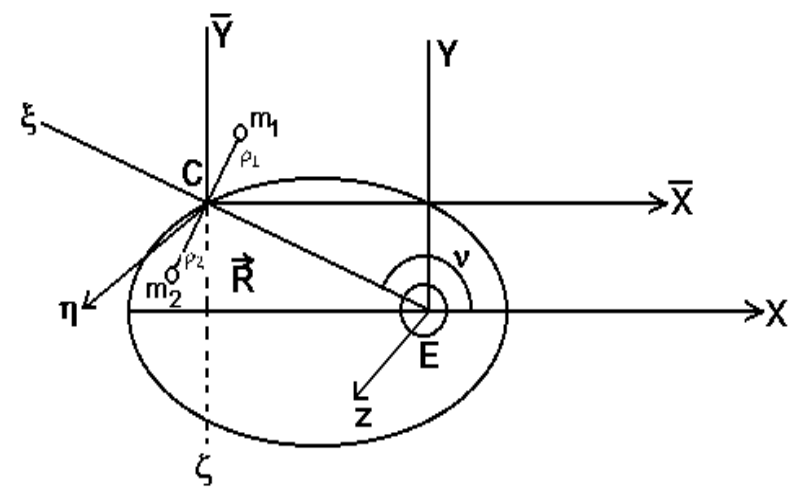

Fig. 1: Rotating Frame of Reference

Here $x$-axis is in the direction of position vector joining the centre of mass of the system and the attracting centre and the $y-$ axis is along the normal to the position vector in the orbital plane of the centre of mass in the direction of the motion of the satellite $m_{1}$ where $\mathrm{A}$ is the Oblateness due to the Earth and $\mathrm{B}$ is the magnetic field of the Earth.

Moreover:

$$
\begin{aligned}
& A=\frac{3 k_{2}}{\rho^{2}} ; \quad \lambda_{\alpha}=\frac{p^{3} \rho^{4}}{\mu}\left[\frac{m_{1}+m_{2}}{m_{1} m_{2}}\right] \lambda ; \\
& B=-\left(\frac{m_{2}}{m_{1}+m_{2}}\right)\left[\frac{Q_{1}}{m_{1}}-\frac{Q_{2}}{m_{2}}\right] \frac{\mu_{E}}{\sqrt{\mu p}} ; \\
& \rho=\frac{R}{p}=\left(\frac{1}{1+e \cos \mathrm{v}}\right) .
\end{aligned}
$$

The dipole of the Earth has its axis inclined from the polar axis of the Earth by a value of $1104^{\prime}$. The angle $\phi$ and $\Omega$ completely define the position of $k_{e}$, unit vector along the axis of magnetic dipole of the Earth.
In this case the condition for constrained is given by the in equality:

$$
x^{2}+y^{2} \leq \frac{1}{\rho^{2}}
$$

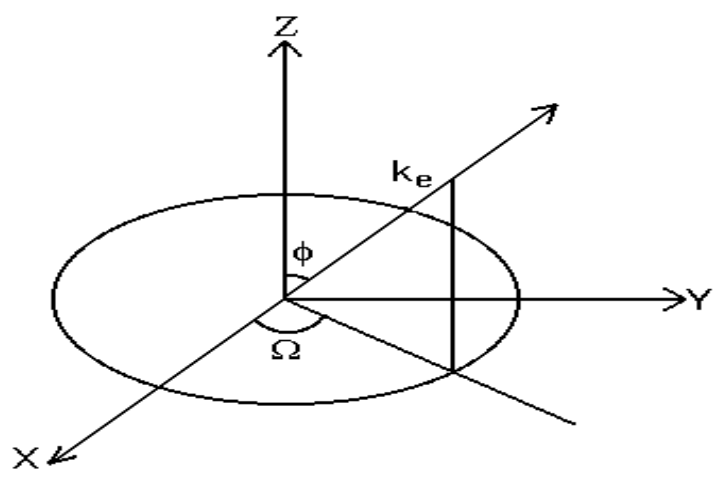

Fig. 2: Orientation of $k_{e}$

Where $\lambda$ denotes Lagrange's multiplier and $\mu$ denotes product of the gravitational constant and the mass of the Earth, where:

$Q_{1}=\left[\frac{\text { Charge } \mathrm{q}_{\mathrm{i}} \text { of the } i^{\text {th }} \text { particle }}{\text { Velocity of light }}\right] i=1,2$, On the masses $m_{1}$ and $m_{2}$, where $\mathrm{v}$ is the true anomaly of the centre of mass of the system in elliptical orbit.

And $\rho=\frac{R}{p}=\left(\frac{1}{1+e \cos \mathrm{v}}\right)$;

Where $P$ and $e$ are the focal parameter and the eccentricity of the orbit of the centre of mass. In equation(2.1), the prime denotes differentiation with respect to $\mathrm{v}$. When the motion of the satellite $m_{1}$ of the system is determined with the help of equation (2.1), the motion of the satellite $m_{2}$ is easily determined with the help of identity.

$$
m_{1} \vec{\rho}_{1}+m_{2} \vec{\rho}_{2}=0
$$

Where $\stackrel{\mathrm{I}}{\rho}_{1}$ and $\stackrel{\mathrm{I}}{\rho}_{2}$ are the radius vectors of the satellites of masses $m_{1}$ and $m_{2}$ respectively with respect to the centre of mass of the system.

Obviously, the actual motion of the system will be combination of three types of motion.

i) Free motion, i.e., $\lambda_{\alpha}=0$.

ii) Constrained motion, i.e., $\lambda_{\alpha} \neq 0$.

iii) Evolutional motion (the combination of free and constrained motion).

We only interested in the constrained motion because free motion are bound to be converted into constrained motion with the lapse of time. In case of constrained motion the equality sign holds in the equation (2.3), i.e.; the particle is moving along the circle of variable radius given by:

$x^{2}+y^{2}=\frac{1}{\rho^{2}}$.

In order to discuss the non-linear planar oscillations of the system, we transform the equation (2.1), into polar form by substituting:

$x=(1+e \cos \mathrm{v}) \cos \psi, ; y=(1+e \cos \mathrm{v}) \sin \psi$.

Where $\psi$ is the angular deviation of the line joining the satellite with the stable position of equilibrium? Solving with respect to $\psi$ and $\lambda_{\alpha}$; we obtain: 
$(1+e \cos \mathrm{v}) \psi^{\prime \prime}-2 e \sin \psi^{\prime}+3 \sin \psi \cdot \cos \psi$

$+5 A(1+e \cos v)^{2} \sin \psi \cdot \cos \psi$

$=B \cos \delta(1+e \cos \mathrm{v}) \sin \psi-B \cos \delta \cdot \sin \mathrm{v} \cdot \cos \psi$

$+2 e \sin \mathrm{v}$.

The equation (2.6) is the equation of motion of a dumbbell satellite in the central gravitational field of the Earth under the influence of the Earth magnetic field and oblateness of the Earth. The equation determining the Lagrange's multiplier is given by:

$$
\begin{aligned}
& (1+e \cos \mathrm{v})^{4}\left(\psi^{\prime}+1\right)^{2}+(1+e \cos \mathrm{v})^{3}\left(3 \cos ^{2} \psi-1\right) \\
& -B \cos \delta(1+e \cos \mathrm{v})^{3}\left[\cos \psi+e \cos (\psi+\mathrm{v})-=\lambda_{\alpha} \geq 0\right. \text {. } \\
& \left.A(1+e \cos \mathrm{v})^{3}\left(4 \cos ^{2} \psi-\sin ^{2} \psi\right)\right]
\end{aligned}
$$

(2.7) the non-linear oscillations described by (2.6) take place as long as inequality given below is satisfied.

$$
\begin{aligned}
& (1+e \cos \mathrm{v})^{4}\left(\psi^{\prime}+1\right)^{2}+(1+e \cos \mathrm{v})^{3}\left(3 \cos ^{2} \psi-1\right) \\
& -B \cos \delta(1+e \cos \mathrm{v})^{3}[\cos \psi+e \cos (\psi+\mathrm{v})- \\
& \left.A(1+e \cos \mathrm{v})^{3}\left(4 \cos ^{2} \psi-\sin ^{2} \psi\right)\right] \geq 0 .
\end{aligned}
$$

Where $\mathrm{v}$ and e are respectively true anomaly of the centre of mass of the system and the eccentricity of the orbit of the system. The prime denotes differentiation with respect to true anomaly v. The system of equation(2.1) oscillates about the stable position of equilibrium in which it lies wholly along the radius vector joining the centre of mass and the centre of force Narayan et al [18], [19], [20]. Substituting $2 \psi=\eta$, the equation(2.1) can be expressed as follows:

$$
\begin{aligned}
& \eta^{\prime \prime}+3 \sin \eta=4 e \sin \mathrm{v}+2 e \eta^{\prime} \sin \mathrm{v} \\
& -5 A(1+e \cos v)^{2} \sin \eta-\eta^{\prime \prime} e \cos \mathrm{v} \\
& +2 B \cos \delta \sin \frac{\eta}{2}+2 e B \cos \delta \sin \left(\eta-\frac{v}{2}\right) .
\end{aligned}
$$

Equation (2.9) describes non-linear oscillations of the system in elliptical orbit in the central gravitational field of the oblateness of the Earth together with the Earth magnetic field.

\section{Non-linear non-resonance oscillations of the system about the position of equilibrium for small eccentricity}

The non-linear oscillations of the system of cable-connected satellites under the influence of above mentioned forces described by equation (2.9) will be investigated for non-resonance cases.

$$
\eta^{\prime \prime}+\omega^{2} \eta=e\left[\begin{array}{l}
\beta(\eta-\sin \eta)+2 \eta^{\prime} \sin v+4 \sin v-\eta^{\prime \prime} \cos v \\
+2 B \cos \delta \sin \frac{\eta}{2}-5 A \sin \eta
\end{array}\right]
$$

$+e^{2}\left[10 A \cos v \sin \eta+2 B \cos \delta \sin \left(v-\frac{\eta}{2}\right)\right]$

In the equation (3.1), $\omega^{2}=3$, and $\beta=\left(\frac{\omega^{2}}{e}\right)$, Moreover the nonlinearity $(\eta-\sin \eta)$, will be assumed to be the order of e.

The system described by equation (3.1), moves under the forced vibration due to the presence of the magnetic field of the Earth and oblaeness of the Earth. We are benefited of the smallness of the eccentricity ' $\mathrm{e}$ ' in equation (3.1), and hence solution may be obtained by exploiting the Bogoliubov, Krilov and Mitropolskey method (1961). For e $=0$, the generating solutions of zeroth order are:

$\eta=a \cos \theta ; \quad \theta=\omega \mathrm{v}+\theta^{\phi}$.

Where the amplitude 'a' and phase $\theta^{\phi}$ are constant, which can be determined by the initial conditions. The solution of equations (3.1) is obtained in the form:

$\eta=a \cos \theta+e u_{1}(a, \theta, \mathrm{v})+e^{2} u_{2}(a, \theta, \mathrm{v})+\ldots \ldots .$.

Where the amplitude ' $\mathrm{a}$ ' and phase ' $\theta$ ' are determined by the differential equations.

$\frac{d a}{d \mathrm{v}}=e A_{1}(a)+e^{2} A_{2}(a) \ldots \ldots \ldots$

$\frac{d \theta}{d \mathrm{v}}=\omega+e B_{1}(a)+e^{2} B_{2}(a)$.

From (3.2), we find $\frac{d \eta}{d \mathrm{v}}$ and $\frac{d^{2} \eta}{d \mathrm{v}^{2}}$ then substituting the value of $\eta, \frac{d \eta}{d \mathrm{v}}$ and $\frac{d^{2} \eta}{d \mathrm{v}^{2}}$, in equation (3.1), and equating the coefficients of like powers of 'e' we get:

$\omega^{2} \frac{\partial^{2} u_{1}}{\partial \theta^{2}}+2 \omega \frac{\partial^{2} u_{1}}{\partial \theta \partial \mathrm{v}}+\frac{\partial^{2} u_{1}}{\partial \mathrm{v}^{2}}-2 \omega A_{1} \cos \theta$

$-2 \omega B_{1} \sin \theta+\omega^{2} u_{1}=$

$\left[4 \sin v+2 \eta^{\prime} \sin v+\beta(\eta-\sin \eta)-\eta^{\prime \prime} \cos \mathrm{v}\right.$

$\left.+2 B \cos \delta \cdot \sin \left(\frac{\eta}{2}\right)+5 A \sin \eta\right]$.

$\omega^{2} \frac{\partial^{2} u_{2}}{\partial \theta^{2}}+2 \omega \frac{\partial^{2} u_{2}}{\partial \theta \partial \mathrm{v}}+\frac{\partial^{2} u_{2}}{\partial \mathrm{v}^{2}}+\omega^{2} u_{2}=$

$\left[-5 A \cos \mathrm{v} \sin \eta+2 \mathrm{~B} \cos \delta \cos v \sin \frac{\eta}{2}-2 \mathrm{~B} \cos \delta \sin \frac{\eta}{2}\right]$

$+A_{1} a \sin \theta \frac{\partial B_{1}}{\partial a}-A_{1} \cos \theta \frac{\partial A_{1}}{\partial a}-2 \omega B_{1} \sin \theta \frac{\partial^{2} u_{1}}{\partial \theta^{2}}$

$+a \cos \theta\left(B_{1}^{2}+2 \omega B_{2}\right)-2 \omega A_{1} \frac{\partial^{2} u_{1}}{\partial \mathrm{a} \partial \theta}$

$+2 \sin \theta\left(\mathrm{A}_{1} B_{1}+2 \omega A_{2}\right)-2 B_{1} \frac{\partial^{2} u_{1}}{\partial \theta \partial \mathrm{v}}-2 A_{1} \omega \frac{\partial^{2} u_{1}}{\partial \mathrm{a} \partial v}$

$-\beta u_{1} a \cos \theta+2 \omega A_{2} \sin \theta+2 a \omega B_{2} \cos \theta$.

Using Fourier expansion given by

$\sin (a \cos \theta)=2 \sum_{k=0}^{\infty}(-1)^{k} \cdot J_{2 k+1}(a) \cdot \cos (2 k+1) \theta$

$\cos (a \cos \theta)=J_{0}(a)$

$+2 \sum_{k=0}^{\infty}(-1)^{k} \cdot J_{2 k}(a) \cdot \cos 2 k \theta$.

Where $J_{k}, k=01,2,3 \ldots \ldots .$. stands for Bessel's function. Substituting these values in equation (3.5) and determining $A_{1}(a)$ and $B_{1}(a)$ in such a way as $u_{1}(a, \theta, \mathrm{v})$, should not contain resonance terms and hence, equating the coefficients of $\sin \theta$ and $\cos \theta$ to zero, separately, we obtain:

$A_{1}(a)=0 ; B_{1}(a)=\left[\begin{array}{l}-\frac{\beta a}{2 \omega}+\frac{10 A J_{1}(a)}{2 \omega}-\frac{\beta}{\omega} J_{1}(a) \\ +\frac{B \cos \delta}{\omega} J_{1}\left(\frac{a}{2}\right)\end{array}\right]$ 
With the help of the equation (3.8) it is not difficult to obtain $u_{1}(a, \theta, v)$ in the form:

$$
\begin{aligned}
& u_{1}(a, \theta, \mathrm{v})=\frac{4 \sin \mathrm{v}}{\left(\omega^{2}-1\right)}-\frac{3 a \cos (\mathrm{v}+\theta)}{2(2 \omega+1)}-\frac{a \cos (\mathrm{v}-\theta)}{2(2 \omega-1)} \\
& +\frac{B}{2 k(k+1)} \sum_{k=1}^{\infty}(-1)^{k} J_{2 k+1}(a) \cos (2 k+1) \theta \\
& -\frac{5 A}{2 k(k+1)} \sum_{k=1}^{\infty}(-1)^{k} J_{2 k+1}(a) \cdot \cos (2 k+1) \theta \\
& -\frac{B \cos \delta}{2 k(k+1)} \sum_{k=1}^{\infty}(-1)^{k} J_{2 k+1}\left(\frac{a}{2}\right) \cdot \cos (2 k+1) \theta .
\end{aligned}
$$

In order to obtain the second approximation of the solution, we need to determine $A_{2}(a)$ and $B_{2}(a)$, and $u_{1}(a, \theta, \mathrm{v})$ as obtained in (3.8) and (3.9), in equation (3.6), and equating the coefficients of $\sin \theta$ and $\cos \theta$ to zero with a view to eliminate resonance terms from $u_{2}(a, \theta, \mathrm{v})$, we obtain:

$A_{2}(a)=0$;

$B_{2}(a)=\left[\begin{array}{l}-\frac{\beta^{2} a^{2}}{4 \omega^{3}}+\frac{20 A \beta J_{1}(a)}{4 \omega^{3}}-\frac{2 \beta a J_{1}(a)}{2 \omega^{2}} \\ +\frac{2 B a \cos \delta}{2 \omega^{3}} J_{1}\left(\frac{a}{2}\right)-\frac{20 A \beta J_{1}(a)}{2 \omega^{3}} \\ -\frac{20 A J_{1}(a) B \cos \delta}{2 \omega^{3}} J_{1}\left(\frac{a}{2}\right)\end{array}\right]$

Thus, in the second approximation, the solution is given by: $\eta=a \cos \theta+e u_{1}(a, \theta, v)$;

Where the amplitude 'a' and phase ' $\theta$, are given by:

$\frac{d a}{d \mathrm{v}}=e A_{1}(a)+e^{2} A_{2}(a) ;$

$\frac{d \theta}{d \mathrm{v}}=\omega+e B_{1}(a)+e^{2} B_{2}(a)$.

And in the second approximation the solution is obtained as:

$$
\begin{aligned}
& \eta=a \cos \theta+\frac{4 \sin \mathrm{v}}{\left(\omega^{2}-1\right)}-\frac{3 a \cos (\mathrm{v}+\theta)}{2(2 \omega+1)}-\frac{a \cos (\mathrm{v}-\theta)}{2(2 \omega-1)} \\
& +\frac{B}{2 k(k+1)} \sum_{k=1}^{\infty}(-1)^{k} J_{2 k+1}(a) \cos (2 k+1) \theta \\
& -\frac{5 A}{2 k(k+1)} \sum_{k=1}^{\infty}(-1)^{k} J_{2 k+1}(a) \cdot \cos (2 k+1) \theta \\
& -\frac{B \cos \delta}{2 k(k+1)} \sum_{k=1}^{\infty}(-1)^{k} J_{2 k+1}\left(\frac{a}{2}\right) \cdot \cos (2 k+1) \theta .
\end{aligned}
$$

Where the amplitude 'a' and phase ' $\theta$ ' are given by:

$$
\begin{aligned}
& \frac{d a}{d \mathrm{v}}=0 \\
& \text { i.e., } a=a_{0} \text { Constant }
\end{aligned}
$$

$$
\begin{gathered}
\frac{d \theta}{d \mathrm{v}}=\omega+e\left[\begin{array}{l}
-\frac{\beta a}{2 \omega}+\frac{10 A J_{1}(a)}{2 \omega} \\
-\frac{\beta}{\omega} J_{1}(a)+\frac{B \cos \delta}{\omega} J_{1}\left(\frac{a}{2}\right)
\end{array}\right]+ \\
e^{2}\left[\begin{array}{l}
-\frac{\beta^{2} a^{2}}{4 \omega^{3}}+\frac{20 A \beta J_{1}(a)}{4 \omega^{3}}-\frac{2 \beta a J_{1}(a)}{2 \omega^{2}} \\
+\frac{2 B a \cos \delta}{2 \omega^{3}} J_{1}\left(\frac{a}{2}\right)-\frac{20 A \beta J_{1}(a)}{2 \omega^{3}} \\
-\frac{20 A J_{1}(a) B \cos \delta}{2 \omega^{3}} J_{1}\left(\frac{a}{2}\right)
\end{array}\right]
\end{gathered}
$$

$\theta=\omega v+\theta^{*}$ Where $\theta^{*}$ is constant.

From the above solution, we concluded that amplitude ' $a$ ' remain constant up to the order of the square of the eccentricity. The phase of the oscillations of the system in this case of non-linear, non-resonance oscillations varies with respect to true anomaly. However the variation of the phase is of the order of the square of the eccentricity, which is a small quantity. We arrived at the conclusion that the system has main resonance at $\omega= \pm 1$, and parametric resonances at $\omega= \pm \frac{1}{2}, \omega= \pm \frac{1}{4}$ for these values of $\omega$, the solution fails as we get singularity. The parametric resonance at $\omega= \pm \frac{1}{4}$ arises due to non-linearity condition.

\section{Non-linear parametric driven oscillations of dumbell satellite system about the position of equilibrium for small eccentricity}

The non-linear oscillations of the dumbell satellite under the influence of the above mentioned

Forces described by (2.3), will be investigated for the parametric resonance case on the assumption

That magnetic field parameter is of the order ' $e$ ' then, equation (2.3), can be put in the form:

$$
\begin{aligned}
& \eta^{\prime \prime}+\omega^{2} \eta=e\left[\begin{array}{l}
\beta(\eta-\sin \eta)+2 \eta^{\prime} \sin v+4 \sin v \\
-\eta^{\prime \prime} \cos v+2 B \cos \delta \sin \frac{\eta}{2}-5 A \sin \eta
\end{array}\right] \\
& +e^{2}\left[10 A \cos v \sin \eta+2 B \cos \delta \sin \left(v-\frac{\eta}{2}\right)\right]
\end{aligned}
$$

Where $\omega^{2}=3$, and $\beta=\left(\frac{\omega^{2}}{e}\right)$. More over the non-linearity term $(\eta-\sin \eta)$ will be assumed to be the order of e.

The system described by equation (4.1), moves under the forced vibration due to the presence of the magnetic field of the earth and oblateness of the earth. this periodic sine force of erturbative nature as long as the period of oscillations of the system is different from the period of sine force for which solution is obtains. as the period of sine force is always changing, it may become equal to the sine force, in that case the periodic sine force plays vital role in the oscillatory motion of the system. while examining the nonresonance case, we conclude that the system experience parametric resonance behavior at and near $\omega=\frac{1}{2}$, and hence the nonresonance solution fails. we are benefitted of the smallness of the eccentricity ' $\mathrm{e}$ ' in equation (3.1), and hence the solution of the differential equation may be obtained by exploiting the bogoliubov, krilov and metropoloskey method.we constructs the asymptotic solutions of the system representing (4.1), in the most 
general case, which is valid at and near the main resonance $\omega=\frac{1}{2}$, exploiting the well known bogoliubov, krilov and metropoloskey, method. the solution of equation (4.1), in the first approximation will be sought in the form:

$\eta=a \cos \left(\frac{v}{2}+\theta\right)$

$\frac{d a}{d \mathrm{v}}=e A_{1}(a, \theta)$;

$\frac{d \theta}{d \mathrm{v}}=\left(\omega-\frac{1}{2}\right)+e B_{1}(a, \theta)$

Where $A_{1}(a, \theta)$ and $B_{1}(a, \theta)$ are particular solution periodic with respect to ' $\theta$ ' of the system.

$\left(\omega-\frac{1}{2}\right) \frac{\partial A_{1}}{\partial \theta}-2 a \omega B_{1}=\frac{1}{2 \pi^{2}} \sum_{\sigma=-\infty}^{\sigma=+\infty} e^{-2 i \sigma \theta} ;$

$\int_{0}^{2 \pi} \int_{0}^{2 \pi} f\left(a, \eta, \eta^{\prime}, \cdot \eta^{\prime \prime}\right) e^{-2 i \sigma \theta^{\prime}} \cos k d \mathrm{v} d k$

$a\left(\omega-\frac{1}{2}\right) \frac{\partial B_{1}}{\partial \theta}+2 \omega A_{1}=-\frac{1}{2 \pi^{2}} \sum_{\sigma=-\infty}^{\sigma=+\infty} e^{-2 i \sigma \theta}$

$\int_{0}^{2 \pi} \int_{0}^{2 \pi} f\left(a, \eta, \eta^{\prime}, \eta^{\prime \prime}\right) e^{-2 i \sigma \theta^{\prime}} \sin k d \mathrm{v} d k$

Where $\theta=k-\frac{v}{2}=\theta^{\prime}$ and $f\left(a, \eta, \eta^{\prime}, \eta^{\prime \prime}\right)$, is the coefficient of 'e' on the right hand side of equation (3.1).

$$
f\left(a, \eta, \eta^{\prime}, \eta^{\prime \prime}\right)=e\left[\beta(\eta-\sin \eta)+2 \eta^{\prime} \sin \mathrm{v}+4 \sin \mathrm{v}\right.
$$

Where

$$
\left.-\eta^{\prime \prime} \cos \mathrm{v}+2 B \cos \delta \cdot \sin \frac{\eta}{2}-5 A \sin \eta\right] \text {. }
$$

Simple integration gives us:

$$
\begin{aligned}
& \left(\omega-\frac{1}{2}\right) \frac{\partial A_{1}}{\partial \theta}-2 a \omega B_{1}=\beta\left(a-2 J_{1}(a)\right) \\
& -10 A J_{1}(a)+4 B \cos \delta J_{1}\left(\frac{a}{2}\right)-\frac{a}{2} \cos 2 \theta \\
& a\left(\omega-\frac{1}{2}\right) \frac{\partial B_{1}}{\partial \theta}+2 \omega A_{1}=\frac{a}{2} \sin 2 \theta .
\end{aligned}
$$

Where $J_{1}(a)$ is the Bessel function of the first order?

$$
\begin{aligned}
& \sin (a \cos \theta)=2 \sum_{n=0}^{\infty}(-1)^{n} \cdot J_{2 n+1}(a) \cdot \cos (2 n+1) \theta \\
& \cos (a \cos \theta)=J_{0}(a)+2 \sum_{n=0}^{\infty}(-1)^{n} \cdot J_{2 n}(a) \cdot \cos 2 n \theta .
\end{aligned}
$$

Where $J_{n}, n=01,2,3 \ldots \ldots .$. stands for Bessel's function.

The periodic solution of the system given by equations (4.6) can obtain as:

$$
\begin{aligned}
& A_{1}=\left[\frac{a \sin 2 \theta}{2}\right] ; \\
& B_{1}=-\frac{\beta}{2 a \omega}\left[\begin{array}{l}
\left(a-2 J_{1}(a)-10 A J_{1}(a)\right. \\
-4 B \cos \delta J_{1}\left(\frac{a}{2}\right)
\end{array}\right]+\frac{\cos 2 \theta}{2} .
\end{aligned}
$$

Where the amplitude ' $a$ ' and phase ' $\theta$ ' are the given by the system of differential equations:

$$
\frac{d a}{d \mathrm{v}}=\frac{e a \sin 2 \theta}{2} \text {; }
$$

$\frac{d \theta}{d \mathrm{v}}=\left(\omega-\frac{1}{2}\right)-\frac{e \beta}{2 a \omega}\left[\begin{array}{l}\left(a-2 J_{1}(a)\right) \\ -10 A J_{1}(a)-4 B \cos \delta J_{1}\left(\frac{a}{2}\right)\end{array}\right]$

$+\frac{e \cos 2 \theta}{2}$

The system of equation can be written as:

$\frac{\partial a}{\partial v}=\frac{1}{a} \frac{\partial H}{\partial \theta}$
$\frac{\partial \theta}{\partial v}=-\frac{1}{a} \frac{\partial H}{\partial a}$.

$$
\begin{aligned}
& H=\frac{-(\omega-1) a^{2}}{2}+\frac{e a^{4}}{64 \omega} \\
& -\frac{e a^{2} \cos 2 \theta}{4}+\frac{5 A e a^{2}}{4 \omega} \\
& -\frac{5 A e a^{4}}{64 \omega}+\frac{e B \cos \delta a^{2}}{4 \omega} \\
& -\frac{e B \cos \delta a^{4}}{256}
\end{aligned}
$$

Obviously, the system of the equation (4.10) has first integral of the form:

$H=c_{0}^{\prime}$;

This reduced the problem to quadrature. Here $c_{0}^{\prime}$, is the constant of integration. However, it is preferable to analyse the integral urves in the phase plane $(a, \theta)$. In order to plot the integral curves reducing the equation (4.11), in the form:

$$
\begin{aligned}
& {\left[\frac{5 A e}{64 \omega}+\frac{e B \cos \delta}{256}-\frac{e}{64 \omega}\right] a^{4}+} \\
& {\left[\frac{e \cos 2 \theta}{4}+\frac{2 \omega-1}{4}-e B \cos \delta-\frac{5 A e}{4 \omega}\right] a^{2}+C_{0}=0} \\
& \text { Where } C_{0}=(n+1) C_{0}^{\prime}
\end{aligned}
$$

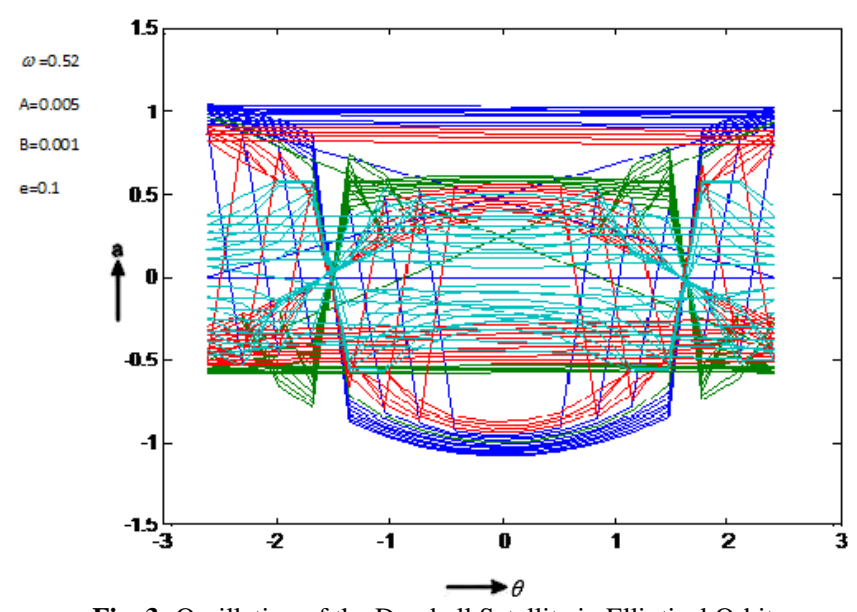

Fig. 3: Oscillation of the Dumbell Satellite in Elliptical Orbit

The integral curves (4.13), have been plotted in Figure (3), for $\omega$ $=0.52, \mathrm{e}=.1, \mathrm{~A}=0.005$ and $\mathrm{B}=0.001$. The integral curves drawn in the phase plane $(a, \theta)$ Using MATLAB software 6.1 versions. It indicates about three zones,one is stationary,allowable and forbidden region and it also indicated that there exists only two stationary regime of the amplitude and it is stable as the integral curves are closed curves.

For any other initial condition we shall obtain periodic change in the amplitude ' $a$ ', which would be bounded. But the maximum value of ' $a$ ' in this case will always be greater than its value at the stationary regime. 
Therefore, for the gravity gradient stablisation of such a space system in elliptical orbit, we are required to bring the amplitude of oscillations near the stationary regime, which gives the smallest deflection of the system from the relative equilibrium position in comparison to any other regime of oscillations.

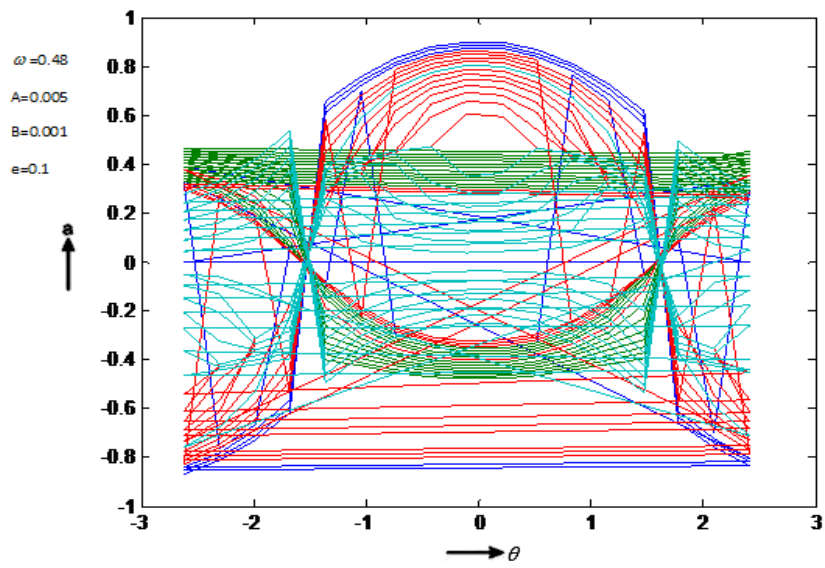

Fig. 4: Oscillations of the Dumbell Satellite in Elliptical Orbit

In the Figure (4), the integral curves for $\omega=0.48$, e $=0.1, \mathrm{~A}=$ 0.005 and $\mathrm{B}=0.001$ has been plotted and it is found that the stationary regime of the amplitude 'a' exists in addition to allowable and forbidden region. In this case also there exists two stationary regime with a slight change in its position. Minute observation of the signature (3) and (4) suggested concluding that during the evolution of oscillations of the system as it approaches the parametric resonance, the stationary amplitude declines steadily with continuously changing phase.

\section{Conclusion}

We have discussed that the combined effects of the Earth Oblateness and the magnetic field of the Earth on the evolutional and non-evolutional motion of cable connected satellites system, connected by a light, flexible and inextensible cable in the centra gravitational field of the Earth for the elliptical orbit of centre of mass of the system. The satellites are considered as charge material particle. The motion of each of their relative to the centre of mass has been studied. It is assumed that centre of mass moves along Keplerian orbit around oblate Earth in elliptical orbit. It is further assumed that satellites are subjected to absolutely nonelastic impacts as the cable tightened. Throughout our analysis, we assumed that the system moves like a dumbbell satellite. We further discussed the non-linear non-resonant and parametric resonant oscillations of dumbbell satellite about the equilibrium point of the system in elliptical orbit.

The equations of motion have been derived in the required form. The non-resonant oscillations of the problem has been studied with the help of Bogoliubov, Krilov and Metropoloskey, method when the eccentricity ' $\mathrm{e}$ ' of the orbit of centre of mass has been taken as the small parameter for the solution of the system.We arrived at the conclusion that the amplitude of oscillations of the system remains constant up to the second order of the approximation. The phase of oscillations varies with respect to true anomaly, but the rate of change is the function of the square of eccentricity of the orbit of the centre of mass. We also come to the conclusion that the system experience main resonance at $\omega= \pm 1$ and parametric resonances at $\omega= \pm \frac{1}{2}, \omega= \pm \frac{1}{4}$, up to the second order of approximation. We also obtained the general solution of the non-linear oscillatory system based upon Bogoliubov, Krilov and Metropoloskey method which is valid at near of the parametric resonance $\omega=\frac{1}{2}$. the method of phase plane has been applied in order to obtain characteristic of the amplitude and the phase of the oscillatory system. We come to the conclusion that there exists two stationary stable regime of the amplitude for both $\omega<\frac{1}{2}$ and $\omega>\frac{1}{2}$.

However the stationary amplitude declines steadily, when it passes through two values of $\omega$ given by $\omega<\frac{1}{2}$ and $\omega>\frac{1}{2}$.

It has also been established that the system will always move like a dumbbell satellite in the phase

Plane $(a, \theta)$ under consideration.

Thus, the oblateness of the Earth and magnetic field of the Earth will play important role in disturbing the attitude of the system of a dumbell satellite in elliptical orbit

\section{References}

[1] Beletsky, V.V. (1969), about the relative motion of two connected bodies, Kosmichekiya Isseldovania, 827-840, In Russian.

[2] Beletsky, V.V., Levin, E.M. (1993), Dynamics of space tether systems, Advances of the Astronomical Sciences, 83, 267-322.

[3] Beletsky, V.V., Novikova, E.T. (1969), about the relative motion of two connected bodies, Kosmichekiya Isseldovania, 7 377-384, In Russian.

[4] Bogoliubov, N.N., Mitropolosky, Y.A. (1961), asymptotic methods in the theory of non-linear oscillations, Hindustan publishing company Delhi 06.

[5] Celletti, Alessandria, Vladislov, V. Sidorenko. (2008), some properties of dumbbell satellite altitude dynamics, Celest. Mech. \& Dyn. Astro, 105-126.

[6] Das, S.K., Bhattacharya, P.K., Singh, R.B. (1976), Effects of magnetic force on the motion of system of two cable connected satellite in orbit, Proc. Nat. Acad. Sci. India, 287-299.

[7] Khan, Ayub, Goel, Neeti. (2011), Chaotic motion in problem of dumbell satellite, International journal comtemp. Maths sciences vol.6, 299-307.

[8] Krupa, M., Kuha, A., Poth W., Schagrl, M., Stiensl, A., Steiner, W., Treger, H., Wiedermann, G. (2000), Tethered satellite systems: A new concept of space flight, European Journal of Mechanics. A Solid $19-$ Special Issue, S145-S164.

[9] Krupa, M., Poth, W., Schagrl, M., Stiensl, A., Steiner, W., Treger, H., Wiedermann, G. (2006), Modelling, dynamics and central of tethered satellite systems, Non -linear Dynamics, 73-96. http://dx.doi.org/10.1007/s11071-006-0752-z.

[10]Liapunov, A.M. The general problem of stability of motion, Sobrania Sachimediviya, 2, In Russian.

[11]Langbort, Cedric. (2002), Bifurcation of relative equilibria in the main problem of artificial satellite theory for a prolate orbit, Celestial Mechanics \& Dynamical Astronomy, 369- 385. http://dx.doi.org/10.1023/A:1021185011071.

[12] Markeev, A.P., (2003), Bardin, B.S. On the stability of planar oscillations and rotation of a satellites in circular orbit, Celestial Mechanics \& Dynamical Astronomy, 57-66.

[13]Mishra, A.K., Modi, V. J. (1982), Deponent and retrieval of shuttle supported tethered satellites Journal of Guidance and Central, 5, No. 3 278- 285.

[14]Narayan, A., Singh, R.B. (1987), Non-linear non-resonance oscillation of interconnected satellites system under the solar pressure about the position of equilibrium for small eccentricity, Proc. Nat. Acad. Sci., India, 427-437.

[15]Narayan, A., Singh, R.B. (1990), Non-linear main resonance oscillation of the system of two interconnected satellites under the influence of solar pressure about stable equilibrium for small eccentricity, Proc. Nat. Acad. Sci., India, 307-313.

[16]Narayan, A., Singh, R.B. (1992), Non-linear parametric resonance oscillation of the system of two interconnected satellites under the influence of solar pressure about the stable position of equilibrium, Proc Nat. Acad. Sci., India vol.63 A.

[17]Narayan, A., Srivastav, S., Dewangan, S. (2004), Effects of earth magnetic field on the stability of cable connected satellites system in equatorial orbit, Journal of AMSE modelling B, 45-60. 
[18]Narayan, A., Pandey, M.D. (2010), Condition of free and constrained motion of cable connected satellites system in low altitude orbit, IJPAM Bulgaria, 107-127.

[19]Narayan, A., Pandey, M.D. (2011), Condition of non-linear stability of dumbell satellite in elliptical orbit. IJPAM Bulgaria, 173-194.

[20]Narayan, A., Pandey, M.D., and Narayan, Amitesh. (2012), some nonlinear resonance oscillations of dumbell satellite in elliptical orbit. IJPAM Bulgaria, 931-944.

[21]Nechville's, V. (1926), Surune Nouvelle formed equations differentials due problem restraint elliptique comptc rendus, Acad. Paris, Compt. Rend. 182- 3100.

[22]Palacian, J.F. (2007), Dynamics of a satellites orbiting a planet with an inhomogeneous gravitational field, Celestial Mechanics \& Dynamical Astronomy, 219-249. http://dx.doi.org/10.1007/s10569-007-9078$\underline{5}$.

[23] Sarychev, V.A., Mirer, S.A. (2000), Relative equilibria of a satellites subjected to gravitational and arodynamics torque's, Celestial Mechanics\& Dynamical Astronomy 76, 55-68. http://dx.doi.org/10.1023/A:1008389730047.

[24] Sarychev, V.A., Mirer, S.A. Degtyarev, A.A., Durate, E.K. (2007) Investigation equilibria of a satellite subjected to gravitational and aerodynamic torques, Celestial Mechanics \& Dynamical Astronomy, 267-287. http://dx.doi.org/10.1007/s10569-006-9064-3.

[25] Sharma, S., Narayan, A. (2001), Non-linear oscillation of inter connected satellite system under the combined influence of the solar radiation pressure and dissipative forces general nature, Bull. Astronomical Soc. India, 29.

[26] Sharma, S., Narayan, A. (2002), Effect of solar radiation pressure on the motion and stability of inter connected satellites system in orbit Indian J. Pure Appl. Math., 33, No. 5, 609-623.

[27]Singh, R.B. (1971), Three dimensional motion of two-connected bodies in the central gravitational field of force, In Problem of Guided Motion in Mechanics, Russian Collection, 210-215.

[28]Singh, R.B. (1973), three dimensional motion of a system of two cables connected satellites in orbit, Astronautica Acta, 19, 301-308.

[29] [29] Singh, B.M., Narayan, A., Singh, R.B. (1997), Non-linear parametric resonance oscillation of the system of two interconnected satellites orbiting around an oblate Earth, Proc. Nat. Acad. Sci., India, 67A 45-55.

[30]Singh, B.M., Narayan, A., Singh, R.B. (2001), Non-linear effects in the motion and stability of an inter connected satellites system orbiting around an oblate Earth, Proc. Nat. Acad. Sci., India, 71, 225-235.

[31] Vladislov, V. Sidorenko, Celletti, Alessandra. (2010), A spring mass model of tethered satellites system properties of planar periodic motions, Celestial Mechanics \& Dynamical Astronomy, 209-231.

[32]Narayan, A, Pandey,M.D. (2013), Some Non-linear Parametric Resonance Oscillations Of Dumbell Satellite In Elliptical Orbit" International Journal of Applied Mathematical Research, Germany, Vol.02 No. 02, 200-212. 